\section{TREATMENT OF THE EYE AND LYMPHATIC GLANDS.}

ACTION OF BISULPHURET OF CARBON, \&C.

To the Editor of The Lancet.

SIR,-In October, 1811, I gave an account in your valuable Journal of the action of the vapour of hydrocyanic acid upon diseases of the eye. Since that period I have been engaged in investigating the action of various other bodies on the same organ, and under the same form.

One reason why $I$ did not rest satisfied with the great effects produced by the hydrocyanjc acid was, that its action, like that of all other medicines, decreased in power by continued application, thereby rendering it necessary to have occasional recourse to other medicines, in order to ensure a more speedy recovery. Another reason was, the reluctance of many individuals to submit the eye to the action of so potent a medicine.

The first medicines to which I shall refer, and which $I$ have employed with some success, are the chlorocyanic acid and sulphuretted chyazic acid.

The plan I pursue is that of putting a dracbm of one of the medicines into a bottle (containing a small piece of sponge) of about two-ounce size, having a mouth precisely fitted to the eye, with a ground-glass stopper. The action of these medicines is very different from that of hydrocyanic acid, in so far as they both stimulate the eye and produce much greater warmth and irritation, with less dilatation of the pupil. Few, however, can bear the chlorocyanic acid to be applied longer to the eye than half a minute, though in a minute after its application all irritation is removed, and the eye feels perfectly at ease.

The next medicine which $I$ have employed in the form of vapour was the chloruret of iodine. This medicine produces very little warmth or uneasiness to the eye, if continued for the space of two minutes or upwards, but a sensation of irritation, accompanied by a How of tears, takes place on its removal. It contracts the pupil, and in no case have I seen it dilate it. Its vapour rises very readily, and does not leave the yellow, disagreeable colouring on the skin, that is produced by the vapour of iodine when uncombined, which is a great drawback in the use of iodine in diseases of the eye.

The last medicine which I have employed is the bisulphuret of carbon, which is so volatile that the application of it to the eye, when the bottle is held in a warm hand for a few seconds, is as much as can be borne, in consequence of the intense pricking heat and flow of tears which it occasions. Owing to this fact, I generally use it by causing the patient to shut the eyelid during its application, which can then be continued, for a minute or two, with the same beneficial effect upon the eye, and without inconvenience to the patient. It generally contracts the pupil, and very seldom dilates it.

I used to employ iodine by putting it into the same bottles, and immersing it in hot water, and, in its state of vapour, applying it to the eye, but $I$ now find it answers much better when dissolved in the bisulphuret of carbon.

It is my intention shortly to give a full account of the action of these medicines upon the various forms of disease to which the eye is subject, and also what particular disease each medicine is best calculated to remove. At the same time I shall state such instances of failure as have occurred in my experience, in order that a just estimate may be formed of the value and importance of each medicine.

It may not be out of place here to state, that $I$ have employed with great success the bisulphuret of carbon to enlarged indurated lymphatic glands. In the first instance, I rubbed equal quantities of bisulphuret of carbon and alcohol upon the parts affected, but without any effect upon the glands. But as its effects were so great when its vapour was confined to the eye, I was led to apply it in the form of vapour, and by means of glass bottles, similar to those $\mathbf{I}$ have described. By this means I excluded the action of the medicine from the external air, and thereby prevented its speedy evaporation. When it had been applied about one minute the patient felt the part very cold, but immediately after a gradual heat, accompanied with great prickling, the heat increasing the longer the medicine was kept in contact with the part, until it could no longer be endured.

On removing the glass the part was red to an extent two or three times greater than the part enclosed. In a few days the change in the size of the glands was very great, and by its daily application a complete and speedy removal of the disease was effected. I also find that its action upon diseased glands is more decided, if the surface of the skin is well moistened with water previous to the application of the bottle to the part. The water, in fact, not only prevents the escape of the vapour between the glass and the skin, but assists the imbibition of the carbon; a point of the highest importance, inasmuch as all its action on the part depends upon the exclusion of the atmosphere from the vapour. I may here observe, that these applications occasion no injury whatever to the skin.

I have also found the bisulphuret of carbon and the chlorocyanic acid valuable medicines in the removal of deafness, depending upon a want of nervous energy and deficiency of wax. The mode of its application is substantially the same as that which I employ in diseases of the eye, with this difference only, 
that the bottle is formed with a small neck and stopper, adapted to the size of the orifice of the ear, and held close to the organ, until a considerable degree of warmth is pro. duced.

The action of these medicines which contain so large a share of carbon arises from the carbon in the vapour permeating the cuticle, and coming in contact with the oxygen in the vessels, which is conveyed through every part of the frame by inspiration and otherwise; thereby forming carbonic acid gas, which evolves heat in the ratio of the quantity consumed by the oxygen. The following quotations from the celebrated Liebig, in his work on Organic Chemistry, sufficiently prove the correctness of this position :-

"It is only in those parts of the body to which arterial blood, and with it the oxygen absorbed in respiration, is conveyed, that heat is produced: hair, wool, or feathers, do not possess an elevated temperature."

"This high temperature of the animal body, or, as it may be called, disengage. ment of heat, is uniformly, and under all circumstances, the result of the combination of a combustible substance with oxygen."

"In whatever way carbon may combine with oxygen, the act of combination cannot take place without the disengagement of heat."

"We can no longer doubt that gases of every kind, whether soluble or insoluble in water, possess the property of permeating animal tissues, as water penetrates unsized paper."

Does not the action of medicines containing so large a proportion of carbon which can be brought into contact with the whole external surface of the body, and thereby capable of being easily disengaged, suggest a method of relieving pulmonary disease, that is likely to be attended with no ordinary success, by calling into greater activity the action of the skin, and thereby materially lightening the labour of the lungs? Are we not the more encouraged to expect such re. medial influence in diseases of the lungs, from the fact of its great power in removing indurated glands, which are so common accompaniments of consumption? There can be no doubt of the usefulness of bisulphuret of carbon in skin diseases.

I bave submitted these observations under the conviction that they embody principles which may be carried out and made of great utility to mankind. I am, Sir, your obedient servant,

$$
\text { A. Turnbull, M.D. }
$$

Russell-square, Oct. 20, 1842.

\section{MANNER IN WHICH LIVING ANIMALS GRADUALLY BECOME DEVELOPED.}

The general law of organic formation, although one of the most essential, is at the same time one of the most abstruse branches of anatomy : I shall, therefore, endeavour to point out the advantage to be derived from including in your studies the history of development, by selecting a few of its more obvious applications.

At the present time it is a point of interest not only to the physiologist but to the pathologist, to determine the exact division between the spinal cord and the brain. This may ap. pear a very easy matter to those who regard the spinal marrow as ending at the upper border of the medulla oblongata, and the brain as comprising all the masses placed above that line; but late researches have shown that the true spinal cord extends higher than the superior part of the oblong medulla. These investigations, as well as the earlier and valuable ones of Flourens, when rightly interpreted, tend to fix the limit of the spinal system in the vicinity of the optic thalami. Now, it is a remarkable fact that the celebrated German anatomist, Bär, in his account of the development of the brain, places the true primitive end of the spinal cord at the infundibulum and the optic thalami ; and he further found that the vesicle forming the hemispheres, or true cerebral organ, did not make its appearance till a subsequent period. It thus appears, from the independent inquiries of one of the most profound observers in Europe, that in the early stage of development there is a positive division marked out between the true spinal and the true cerebral systems, accurately corresponding with the conclusion deduced from physiological research. It would be a serions mistake to suppose that these are mere barren speculations in which the practitioner has little or no concern ; on the contrary, by demonstrating that nature herself has marked out, at an early epoch of existence, a structural division between the brain and spinal cord, they give a consistency, amounting almost to certainty, to the views of Dr. Marshall Hall respecting the true seat of convulsive diseases, the importance of which every practitioner who is acquainted with the fearful results of those affections will readily admit.

In another class of cases which will demand your attention in practice, namely, malformations, a knowledge of this branch of anatomy is indispensable. Cases of malformation about the anus, for example, occasionally occur, and you may be called upon to decide on the probable result, and on the propriety of an operation. Now, there are some instances in which the rectum is perfect, the anus being merely closed by the in- 